\title{
Preventive effect of rehabilitation training therapy on muscle quality in patients with stroke. A retrospective study
}

\author{
Dandan Feng ${ }^{\mathrm{a}, \mathrm{b}, \#}$, Can Zhao ${ }^{\mathrm{c}, \#}$, Dan Chen ${ }^{\mathrm{b}}$, Xiaojing He ${ }^{\mathrm{d}}$, Xiang Lu ${ }^{\mathrm{a}, \mathrm{c},},{ }^{*}$, Wei Gao ${ }^{\mathrm{c}, *}$ \\ ${ }^{a}$ School of Public Health, Nanjing Medical University, Nanjing 211166, China. \\ ${ }^{b}$ Department of Rehabilitation Medicine, Sir Run Run Hospital, Nanjing Medical University, Nanjing 211166, China. \\ ${ }^{c}$ Department of Geriatrics, Sir Run Run Hospital, Nanjing Medical University, Nanjing 211166, China. \\ ${ }^{d}$ Department of Health Policy Administration, Nanjing Medical University, Nanjing 211166, China.
}

\begin{abstract}
A retrospective analysis was conducted in 45 patients with stroke. All patients received three weeks of rehabilitation training. Body composition was examined using Bio-impedance analysis methods before and after training. Barthel index was used to evaluate activities of daily living before and after the intervention. After rehabilitation training, total body water, muscle mass, protein content, inorganic salt content, and skeletal muscle content were increased while body fat content and body fat percentage were significantly decreased. Barthel Index scores showed that the ability of daily living was improved after rehabilitation. In conclusion, rehabilitation training therapy may have protective effects on sarcopenia in patients with stroke.

Keywords: Stroke, sarcopenia, rehabilitation training
\end{abstract}

Stroke is a leading cause of mortality and disability. The prevalence of people living with the effects of stroke has increased because of the growing and aging population [1-3]. The most common limb dysfunction after stroke is the weakness of hemiplegia of one side of the limb, which leads to a decline in daily living and serious disability, and further leads to reduction of muscle strength, described as sarcopenia, and strength of patients. Sarcopenia is a common disease in older people [4-7], and is highly prevalent in stroke patients [8-9].

We evaluated the preventive effect of rehabilitation training therapy on post-stroke patients with sarcopenia. A total of 45 patients ( 32 females, 13 males) with cerebral apoplexy and an average age of $56.5 \pm 14.5$ years, who were admitted to the Department of Rehabilitation Medi-

\# These authors contributed equally.

* Corresponding author: Wei Gao

Mailing address: Department of Geriatrics, Sir Run Run Hospital, Nanjing Medical University, Nanjing 211166, China.

Email: gaowei84@njmu.edu.cn

* Corresponding author: Xiang Lu

Mailing address: Department of Geriatrics, Sir Run Run Hospital, Nanjing Medical University, Nanjing 211166, China.

Email: luxiang66@njmu.edu.cn

Received: 20 October 2021 / Accepted: 10 December 2021 cine, Nanjing Medical University, Sir Run Run Hospital, from October 2019 to October 2020 for 3-weeks rehabilitation training, were enrolled in this study. The study was approved by the ethics committee of Nanjing Medical University, Sir Run Run Hospital (ChiCTR1900028296).

The inclusion criteria were: 1) Diagnosis of stroke based on clinical presentations and CT/MRI confirmation; 2) Limb function in patients with left side or both sides hemiplegia obstacle, Brunstrom stage I-VI; 3) Stable vital signs, able to cooperate with rehabilitation training; 4) Able to participate in the experiment voluntarily and to sign the informed consent. The exclusion criteria were: 1) History of malignant tumor; 2) Psychiatric disease, severe cognition impairment, and severe diseases of other systems (patients with severe circulatory system diseases, hematologic system diseases, and immunocompromised functions); 3) Patients with severe limb spasms, modified Ashworth evaluation class acuity II; 4) Patients with cardiac pacemakers or other metal implants; 5) Patients or their family members who were unwilling to cooperate after informing the informed consent.

Discontinuation Criteria: Patients who were unable to continue the rehabilitation training due to other severe acute diseases or cardiovascular and cerebrovascular events.

After initial functional disorder assessment and evaluation of patients by doctors, the rehabilitation training plan 
and schedule were formulated. Exercise training included joint movement training, muscle strength training, balance function training, and walking training. Physical factor therapy included Bioelectrical Feedback Training (BFT) and Neuromuscular electrical stimulation therapy (NES). Occupational therapy included Upper limb intelligent feedback training, Situation simulation training, Activity of daily living (ADL) training. Primary outcome assessments were changes in body composition before and after rehabilitation training and Bio-impedance analysis (InBody S10) to assess body composition before and after the intervention. Secondary outcome assessments were changes in ADL before and after training using the Modified Barthel Index score. Lower scores represent significant deficits and greater dependence of patients [10]. Statistical analysis was conducted using SPSS.26 software. Measurement data were expressed as mean \pm standard deviation, and enumeration data were expressed as a rate (\%). The descriptive analysis method was used to analyze the general data of patients, and comparisons between two groups were assessed with a $t$-test was used to analyze the changes of human index components before and after comprehensive rehabilitation training. The change of ADL scores before and after comprehensive rehabilitation training was analyzed by rank-sum test. $P<0.05$ represented a statistically significant difference.

The results showed that the body composition analysis index of 45 patients had obvious changes after rehabilitation training. The total body moisture, muscle mass, protein content, inorganic salt content, and skeletal muscle content were significantly increased after rehabilitation training, while the body fat content and body fat percentage were significantly decreased $(P<0.05)$. In the analysis of muscle balance of limbs and trunk, the muscle content of limbs and trunk increased after intervention, including right upper limb $(2.02 \pm 0.55$ vs $2.20 \pm 0.69)$, left upper limb $(2.00 \pm 0.56$ vs $2.23 \pm 0.88)$, trunk $(18.37 \pm 3.39$ vs $19.33 \pm 4.22)$, right lower limb $(8.96 \pm 2.14$ vs $9.60 \pm$ $2.12)$, left lower limb $(8.77 \pm 2.05$ vs $9.60 \pm 2.12) . P \leq$ 0.05 ., the water level analysis results of limbs and trunk showed that the water content of limbs and trunk was significantly improved after the rehabilitation training.

Skeletal muscle index (SMI) was significantly increased after training $(P=0.009)$, which proved that rehabilitation training therapy had a significant improvement effect on muscle loss and decline in stroke patients. After rehabilitation training, ICW, ECW, BMR, bone mineral content, somatic cell number, and other body components were significantly improved $(P<0.05)$, at the same time visceral fat area also decreased significantly $(P<0.05)$. The results also showed that there was no statistically significant difference in the changes around the upper arm, suggesting that rehabilitation training did not improve it.

According to the Barthel Index score, 13 patients had mild dysfunction, 6 patients had moderate dysfunction, and 25 patients had severe dysfunction. After the intervention, the numbers were reduced to 22,6 , and 17 . The results of the rank-sum test showed that the daily living ability of the patients was significantly improved after the rehabilitation training, and the difference was statistically significant $(P$ $<0.05)$. This result also confirms the positive preventive effect of rehabilitation training therapy on improving the dysfunction caused by muscle loss in stroke patients.

Several limitations are apparent in our study. First, due to the limit of hospitalization time and economic factors, patients usually cannot stay in the hospital for long periods. Therefore, our rehabilitation training cycle was generally 21 days for one hospitalization cycle. Wang et al. [11 ] reported that short-term exercise has a significant effect on the treatment of sarcopenia in elderly inpatients aged 80 years and above. Even within a short period of two weeks, exercise can effectively improve the daily activity ability of elderly inpatients with sarcopenia recently. Secondly, this study did not refer to and compare the rehabilitation training period of patients, the beginning and end time of training, nutritional supply, and the measurement positions of different patients were different. Third, the relatively small sample size, the inability to randomly assign participants and controls, selection bias, and single-center study design might limit the generalizability of the results. Fourth, as with other clinical trials of rehabilitation training, generalizations of studies may be limited because individual rehabilitation training programs often need to be tailored to their individual circumstances, and there is no guarantee that the training program will be consistent for every patient or group of patients.

In conclusion, our study has presented clinical observations to suggest that rehabilitation training therapy has a positive effect on the prevention of muscle loss and limb function decline after a common stroke. Rehabilitation training therapy can effectively improve muscle content and skeletal muscle quality of stroke patients, improve the ability of daily living, and prevent the symptoms of sarcopenia in stroke patients and the occurrence of sarcopenia after stroke. Rehabilitation training therapy to prevent and treat sarcopenia patients with other disease conditions would be a promising focus and direction for further research.

\section{Declarations}

Authors' contributions: Made substantial contributions to conception and design of the study: Wei Gao, Xiang Lu; Performed data analysis and interpretation: DandanFeng, Can Zhao; Performed data acquisition and technical support: Dan Chen, Xiaojing He.

Availability of data and materials: Not applicable.

Financial support and sponsorship: National Key R\&D Program of China (No.2020YFC2008500) and a Grant from Jiangsu Geriatrics Society (No. JGS2019ZXYY06).

Conflict of interest: The authors declare that they have no conflict of interest.

Ethical approval and consent to participate: This study was performed in accordance with the principles outlined 
in the Declaration of Helsinki and approved by the Ethics Committee of Sir Run Run Hospital, Nanjing Medical University (ChiCTR1900028296). Written informed consent was obtained from each participant.

\section{References}

1. Ciorba A, Aimoni C, Crema L, et al. Sudden hearing loss and the risk of subsequent cerebral ischemic stroke. BENT, 2015, 11(3): 205-209.

2. Bonifačić D, Toplak A, Benjak I, et al. Monocytes and monocyte chemoattractant protein 1 (MCP-1) as early predictors of disease outcome in patients with cerebral ischemic stroke. Wiener klinische Wochenschrift, 2016, 128(1-2): 20-27.

3. Stinear C M, Lang C E, Zeiler S, et al. Advances and challenges in stroke rehabilitation. The Lancet Neurology, 2020, 19(4): 348-360.

4. Cruz-Jentoft A J, Bahat G, Bauer J, et al. Sarcopenia: revised European consensus on definition and diagnosis. Age Ageing, 2019, 48(1): 16-31.

5. Morley J E, Abbatecola A M, Argiles J M, et al. Sarcopenia with limited mobility: an international consensus. Journal of the American Medical Directors Association, 2011, 12(6): 403-409.
6. Chen L K, Liu L K, Woo J, et al. Sarcopenia in Asia: consensus report of the Asian Working Group for Sarcopenia. Journal of the American Medical Directors Association, 2014, 15(2): 95-101.

7. Cadilhac D A, Kim J, Lannin N A, et al. Better outcomes for hospitalized patients with TIA when in stroke units: an observational study. Neurology, 2016, 86(22): 20422048.

8. Yoshimura Y, Wakabayashi H, Bise T, et al. Prevalence of sarcopenia and its association with activities of daily living and dysphagia in convalescent rehabilitation ward inpatients. Clinical Nutrition, 2018, 37(6): 2022-2028.

9. Shiraishi A, Yoshimura Y, Wakabayashi H, et al. Prevalence of stroke-related sarcopenia and its association with poor oral status in post-acute stroke patients: Implications for oral sarcopenia. Clinical Nutrition, 2018, 37(1): 204-207.

10. Liu W, Unick J, Galik E, et al. Barthel Index of activities of daily living: item response theory analysis of ratings for long-term care residents. Nursing research, 2015, 64(2): 88-99.

11. Wang R, Liang Y, Jiang J, et al. Effectiveness of a shortterm mixed exercise program for treating sarcopenia in hospitalized patients aged 80 years and older: A prospective clinical trial. The journal of nutrition, health \& aging, 2020, 24(10): 1087-1093.

Cite this article as: Feng D D, Zhao C, Chen D, et al. Preventive effect of rehabilitation training therapy on muscle quality in patients with stroke. A retrospective study[J]. Aging Pathobiology and Therapeutics, 2021, 3(4): 136-138. 